\title{
CHEMICAL CUES MEDIATING BEHAVIORAL AND ELECTROPHYSIOLOGICAL RESPONSES OF FOPIUS ARISANUS (HYMENOPTERA: BRACONIDAE): THE ROLE OF HERBIVORE- INDUCED PLANT VOLATILES
}

\author{
CAI, P.M. ${ }^{1,2,3}-$ SONG, Y.Z. ${ }^{1}-$ HUO, D. ${ }^{1}-$ LIN, J. ${ }^{2,3}-$ ZHANG, H.M. ${ }^{1}-$ ZHANG, Z.H. ${ }^{1}-$ HUANG, \\ F.M. ${ }^{1}-$ XIAO, C.M. ${ }^{1}-$ JI, Q.E. ${ }^{2,3^{*}}$ \\ ${ }^{1}$ Department of Horticulture, College of Tea and Food Science, Wuyi University, Wuyishan, \\ 354300 Fujian Province, China \\ (e-mails: caipumo@163.com-P.M.Cai; 1023554932@qq.com-Y.Z. Song; \\ hawda1090@163.com-D.Huo; z15159754369@163.com-H.M. Zhang; \\ Zhangzihao1095@163.com-Z.H.Zhang;Ulikexx123@163.com-F.M. Huang; \\ XGM2352033@163.com-C.M.Xiao) \\ ${ }^{2}$ Institute of Beneficial Insects, Plant Protection College, Fujian Agriculture and Forestry \\ University, Fuzhou, 350002 Fujian Province, China \\ (e-mail: Lin14787861578@163.com-J.Lin)
}

${ }^{3}$ Key Lab of Biopesticide and Chemical Biology, Ministry of Education, Fuzhou, 350002 Fujian Province, China

*Correspondence author e-mail: jiqinge@yeah.net; phone: +86-591-8378-9420; fax: +86-591-8378-9421

(Received 25 $5^{\text {th }}$ Mar 2020; accepted $2^{\text {nd }}$ Jul 2020)

\begin{abstract}
The aim of the present study was to establish how a selection of herbivore-induced plant volatiles (HIPVs) regulate the behavioral response of Fopius arisanus. A total of ten HIPVs were selected which are major bioactive components produced by fruits immediately after being infested with eggs of Bactrocera dorsalis, a common pest parasitized by $F$. arisanus. The behavioral and electroantennogram (EAG) responses of both sexes of $F$. arisanus to these chemicals were investigated. Our results suggest that all tested HIPVs can elicit different levels of antennal and behavioral response for both sexes at a variety of concentrations. There were no significant differences in response levels between sexes for all compounds, except trans farnesol at a concentration of $10^{-2} \mathrm{ml} / \mathrm{ml}(\mathrm{v} / \mathrm{v})$, indicating that it may be of value in biological control programs using $F$. arisanus. Furthermore, we observed that the behavioral and EAG dose-dependent curves of both sexes of $F$. arisanus could be largely divided into four types, namely steady decrease (ethyl octanoate, methyl octanoate, linalool, tetradecane, pentadecane), steady increase (trans farnesol), inverted V (cis-3-hexenyl acetate, $\beta$-myrcene, $\beta$-ocimene) and fluctuating (benzaldehyde). The ecological role of these HIPV components in the host location process of $F$. arisanus is discussed.
\end{abstract}

Keywords: HIPVs, biological control, EAG response, behavioral responses, infochemicals

\section{Introduction}

Within the context of biological control programs, the effectiveness of a parasitoid relies heavily on its capacity to search for suitable hosts (Godfray, 1994). Insects have developed multisensory systems for perceiving various cues from their habitats, including visual, chemical and vibrational cues, which are utilized in an interactive manner to make foraging choices (Schellhorn et al., 2014). Of these, chemical cues play the most important role and influence activities such as food finding, mate searching and escaping natural enemies and competition. These, in turn, affect the large-scale 
geographical distribution of insect populations (Schoonhoven et al., 2005; Vinatier et al., 2011). Chemical cues from either the phytophagous host or its habitat may be used by parasitoids at long- and short-range. Generally, cues derived from the host are less detectable but a more reliable indication of the presence of herbivorous hosts. Cues from the host plant, on the other hand, are less reliable but more detectable, which presents the parasitoid with a reliability-detectability dilemma (Vet and Dicke, 1992).

Parasitoids have developed diverse tactics to overcome this predicament, one of which is the utilization of herbivore-induced plant volatiles (HIPVs) as reliable cues to find their herbivorous hosts in a complex odorous environment. This also has the indirect effect of defending host habitats from infestation (Vet and Dicke, 1992; NgiSong et al., 2000). These volatiles usually comprise hundreds of chemical components distributed within the green leaf volatiles, and include esters, aromatic compounds, monoterpenes, sesquiterpenes and homoterpenes (Degenhardt et al., 2009); however, not all components of HIPVs trigger behavioral responses in parasitoids. In some tritrophic contexts, single critical compounds only or a blend of compounds with specific ratios have been found to affect the recruitment and recognition of insects (Bruce and Pickett, 2011). This indicates that it is necessary to investigate how parasitoids respond to volatile infochemicals for each parasitoid-host system and to characterize which infochemicals are involved.

The subject of the present research is Fopius arisanus Sonan (Hymenoptera: Braconidae), an egg-pupal endoparasitoid originating in the Indo-Pacific region and known to parasitize the eggs and first instars of approximately 40 frugivorous tephritid fruit fly species (Bautista and Harris, 1996; Rousse et al., 2007a). This parasitoid was introduced into Hawaii at the end of the 1940s and into French Polynesia later in 2002; in both cases it rapidly became the dominant parasitoid of tephritid pests, greatly suppressing the populations of Bactrocera dorsalis Hendel and Ceratitis capitata Wiedemann (Diptera: Tephritidae), respectively (Vargas et al., 2007, 2012, 2013). These cases of successful application demonstrated that $F$. arisanus possessed a huge potential for augmentative biological control, alone or in combination with other management measures to control fruit flies (Harris et al., 2000).

The biology of $F$. arisanus is well-known, and its host-searching behavior has been reported on coffee fruits infested by $C$. capitata eggs (Wang and Messing, 2003). Furthermore, many studies have investigated the relationships between fly-infested plants and the ovipositional behavior or parasitism performance of parasitoids (Liquido, 1991; Harris and Bautista, 1996; Bautista et al., 2004; Ayelo et al., 2017). Additionally, F. arisanus was fascinated with fresh guava and orange fruits (Altuzar et al., 2004), and infestation by Anastrepha eggs enhanced this attraction (Rousse et al., 2007b; Pérez et al., 2013). In recent years, our research team has analyzed the volatile components of four host crops, namely guava (Psidium guajava L. [Myrtales: Myrtaceae], banana (Musa paradisiaca L. [Zingiberales: Musaceae]), citrus (Citrus sinensis L. [Sapindales: Rubiaceae]) and tomato Solanum lycopersicum Mill. [Tubiflorae: Solanaceae], by gas chromatography-mass spectrometry (GC-MS). We found that after egg deposition by $B$. dorsalis, host fruits can emit ten new chemical components in comparison with noninfested fruits ( $\mathrm{Gu}$ et al., 2017). When we compared these volatile compounds with the volatile profile of the egg surface of B. dorsalis (Ji et al., 2016), we found that the emitted compounds derived from the fruits, rather than from the host pest. Until now, the ecological role of HIPVs emitted from fruits after $B$. dorsalis infestation in the hostlocation behavior of $F$. arisanus is poorly understood. 
The purpose of the present research was to construct dose-response curves of key bioactive HIPVs that modulate the tritrophic system using electrophysiological and behavioral assays. Understanding how parasitoids respond to such chemicals will significantly contribute to the current knowledge on insect chemical communication and hopefully improve their effectiveness as biological control agents against destructive fruit pests like B. dorsalis.

\section{Materials and methods}

\section{Parasitoid rearing}

The initial colony of parasitoid $F$. arisanus was obtained from a field culture, reared on B. dorsalis eggs as described by Manoukis et al. (2011). Experimental parasitoids were kept at the insect mass rearing chamber of the Beneficial Insects Institute, Fujian Agriculture and Forestry University (BII, FAFU), Fujian Province, China under conditions of $25 \pm 1{ }^{\circ} \mathrm{C}, 65 \pm 5 \%$ relative humidity $(\mathrm{RH})$, and a L:D photoperiod of 10:14 h. The laboratory-reared strains were periodically introduced with a parasitoid population collected from the field to prevent genetic decline and to preserve the original behavioral properties of the species. Naïve mated parasitoid females and males aged 7-12 days (without previous exposure to host or plant odors) acquired from the fifth to sixth generation were used in the assays. All experiments were conducted between 08:00 and 16:00.

\section{Chemical components}

Ten chemical standards, namely ethyl octanoate, benzaldehyde, methyl octanoate, linalool, tetradecane, pentadecane, cis-3-hexenyl acetate, $\beta$-myrcene, trans farnesol and $\beta$-ocimene, were used in electroantennogram (EAG) and behavioral assays. Information on these standards is listed in Table 1. Each compound was dissolved into n-hexane (purity > 99\%; Beijing solabo Technology Co., Ltd, Beijing, China) and diluted to concentrations of $10^{-1}, 10^{-2}, 10^{-3}, 10^{-4}$ and $10^{-5} \mathrm{ml} / \mathrm{ml}(v: v)$ for the dose-response tests. Diluted standards were maintained in a refrigerator at $4{ }^{\circ} \mathrm{C}$ until required.

Table 1. Details of the ten chemical standards

\begin{tabular}{c|c|c|c|c|c}
\hline Chemicals & Formula & CAS no. & Purity (\%) & Storage condition & Source \\
\hline Benzaldehyde & $\mathrm{C}_{7} \mathrm{H}_{6} \mathrm{O}$ & $100-52-7$ & 98.5 & Room temperature & Shanghai Macklin Biochemical Technology Co., Ltd \\
\hline Ethyl octanoate & $\mathrm{C}_{10} \mathrm{H}_{20} \mathrm{O}_{2}$ & $106-32-1$ & 99 & Room temperature & Shanghai Macklin Biochemical Technology Co., Ltd \\
\hline Methyl octanoate & $\mathrm{C}_{9} \mathrm{H}_{18} \mathrm{O}_{2}$ & $111-11-5$ & 99 & $\begin{array}{c}\text { Room temperature, } \\
\text { dry, sealed }\end{array}$ & Shanghai Macklin Biochemical Technology Co., Ltd \\
\hline Linalool & $\mathrm{C}_{10} \mathrm{H}_{18} \mathrm{O}$ & $78-70-6$ & 98 & Room temperature & Shanghai Macklin Biochemical Technology Co., Ltd \\
\hline Tetradecane & $\mathrm{C}_{14} \mathrm{H}_{30}$ & $629-59-4$ & 98 & Room temperature & Shanghai Macklin Biochemical Technology Co., Ltd \\
\hline Pentadecane & $\mathrm{C}_{15} \mathrm{H}_{32}$ & $629-62-9$ & 98 & Room temperature & Shanghai Macklin Biochemical Technology Co., Ltd \\
\hline $\begin{array}{c}\text { cis-3-hexenyl } \\
\text { acetate }\end{array}$ & $\mathrm{C}_{8} \mathrm{H}_{14} \mathrm{O}_{2}$ & $3681-71-8$ & 98 & Room temperature & Shanghai Macklin Biochemical Technology Co., Ltd \\
\hline$\beta$-myrcene & $\mathrm{C}_{10} \mathrm{H}_{16}$ & $123-35-3$ & 90 & $2-8{ }^{\circ} \mathrm{C}$ & Aladdin reagent (Shanghai) Co., Ltd \\
\hline Trans farnesol & $\mathrm{C}_{15} \mathrm{H}_{26} \mathrm{O}$ & $106-28-5$ & 97 & $2-8{ }^{\circ} \mathrm{C}$ & Shanghai Macklin Biochemical Technology Co., Ltd \\
\hline$\beta$-ocimene & $\mathrm{C}_{10} \mathrm{H}_{16}$ & $13877-91-$ & 90 & Room temperature & Shanghai Macklin Biochemical Technology Co., Ltd \\
\hline
\end{tabular}




\section{Choice measurements}

This bioassay was designed to determine whether different concentrations of HIPVs imposed differing impacts on the behavioral responses of $F$. arisanus. This was done using a six-choice olfactometer as described by $\mathrm{Gu}$ et al. (2018). The test area comprised a release area, an adapting area, a selection area and six 100-ml odor bottles. Humidified and charcoal-filtered air was passed through the test area uniformly at a rate of $200 \mathrm{ml} / \mathrm{min}$. A piece of cotton wool was placed in each odor bottle which was moistened with $200 \mu \mathrm{l}$ of the test component; one concentration was used for each bottle and pure n-hexane served as the control group. Each trial was tested with only one chemical type. Prior to the experiment, $F$. arisanus adults were starved for $1 \mathrm{~h}$ to improve their sensitivity to odor. One group of 30 parasitoids of the same sex were then concomitantly introduced into the release area. Based on pre-observations, most parasitoids need about $30 \mathrm{~min}$ to adapt to this experimental apparatus and then make a "choice". Therefore, after $30 \mathrm{~min}$ the number of parasitoids that stayed at each area for at least $10 \mathrm{~s}$ or trapped in each odor bottle was documented. The response rate was then calculated using the following formula:

$$
R_{\text {response }}=\frac{N_{\text {response }}}{N_{\text {release }}} \times 100
$$

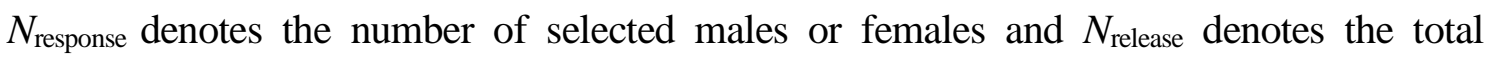
number of released males or females. After each trial, the parasitoids were removed and not used again in the experiment. The apparatus was then cleaned using an abluent and then $75 \%$ ethanol, rinsed using distilled water, and thereafter dried for several hours with an airblowing drier. After each individual trial, different chemicals of various concentrations were randomly deposited into odor bottles to eliminate any positional effects. Six replicates were performed for each six-choice experiment of each chemical type.

\section{Antennal preparation and EAG measurements}

In order to evaluate the dose-dependent effects of each volatile component, the EAG technique was used to determine the electrophysiological responses of the antennae of male and female $F$. arisanus to volatile components at different concentrations. The antennae of $F$. arisanus adults were completely excised with the aid of a stereomicroscope and a few top flagellar segments of the antennae were cut off.

Thereafter, the cut antennal tips were inserted into $\mathrm{Ag}-\mathrm{AgCl}$ glass electrodes filled with saline solution, which was used to maintain an electrical connection between the electrodes. The EAG response signals were passed through a high impedance amplifier (IDIC-2, Syntech, Hilversum, Netherlands) and further processed with EAG software (Syntech).

Different concentrations of the tested components diluted with n-hexane (control) were exposed in ascending order to abate the effect of olfactory adaptation possibly caused by strong stimulation. Stimulus solutions were prepared by first pipetting $20 \mu \mathrm{l}$ of each solution onto individual filter papers $(6 \times 15 \mathrm{~mm})$, and thereafter allowed to adequately diffuse for $2 \mathrm{~min}$ before the assay. The stimuli were pipetted onto filter paper strips and were immediately deposited in disposable Pasteur pipettes that in turn were connected to an air flow control device (Syntech CS-05, Netherlands Syntech Co., Ltd.) which continuously flowed purified and humidified air over the prepared antennae 
at a rate of $100 \mathrm{ml} / \mathrm{min}$. Each air tube was placed $10 \mathrm{~mm}$ from the antenna. The control stimulus containing n-hexane was tested both at the beginning and end of each trial to ensure that the preparations were functional throughout. Five antennae were used for each chemical, and each assay was replicated five times. The electrophysiological response of $F$. arisanus to each chemical was expressed by the EAG relative value $\left(R V_{E A G}\right)$, which was calculated using the following formula:

$$
R V_{E A G}=\frac{V_{1}+V_{2}}{V_{c 1}+V_{c 2}}
$$

$V_{1}$ and $V_{2}$ denote the two EAG values of the test compounds, and $V_{c 1}$ and $V_{c 2}$ denote the two EAG values of the control treatments at the start and the end of each replicate, respectively.

\section{Statistical analysis}

Statistical differences between concentrations for both the behavioral responses and EAG relative values were evaluated using Tukey's honestly significant differences (HSD) test for multiple mean comparisons after ANOVA. A comparison of the differences between male and female parasitoids was conducted using a independent $\mathrm{t}-$ test. In both cases $P<0.05$ was considered statistically significant. All statistical analyses were performed using SPSS 17.0 for Windows (SPSS Inc., Chicago, IL, USA).

\section{Results}

The tendencies of response rates and $E A G$ relative values of $F$. arisanus to ten synthetic HIPVs at different concentrations

Our results show that all dosages of the tested components can elicit different levels of antennal and behavioral response in $F$. arisanus (both male and female). The response rates and EAG relative values of both sexes of $F$. arisanus to the same component showed some significant differences between concentrations (Figs. 1-10; F and $P$ values see Table 2).

The dose threshold that can trigger the statistically highest behavioral and antennal response for each compound is shown in Table 2. It can be seen that both parasitoid females and males have a diverse dose threshold range for different chemicals. Furthermore, for each chemical, there was no difference in trend between males and females for both the response rate and EAG relative values. Benzaldehyde is an exception to this rule, for which the EAG relative values steadily decreased as the dose increased whereas the behavioral response rates of parasitoid males fluctuated with concentration (Fig. 2). The tendencies of the response rates and EAG relative values of $F$. arisanus males and females could be divided into the 4 types described below.

\section{Steady decrease}

Response rates and EAG relative values of both male and female $F$. arisanus declined as the dose increased for ethyl octanoate, methyl octanoate, linalool, tetradecane and pentadecane (Figs. 1 and 3-6). For benzaldehyde, both male and female EAG relative values and female response rates belonged to this type (Fig. 2B). 

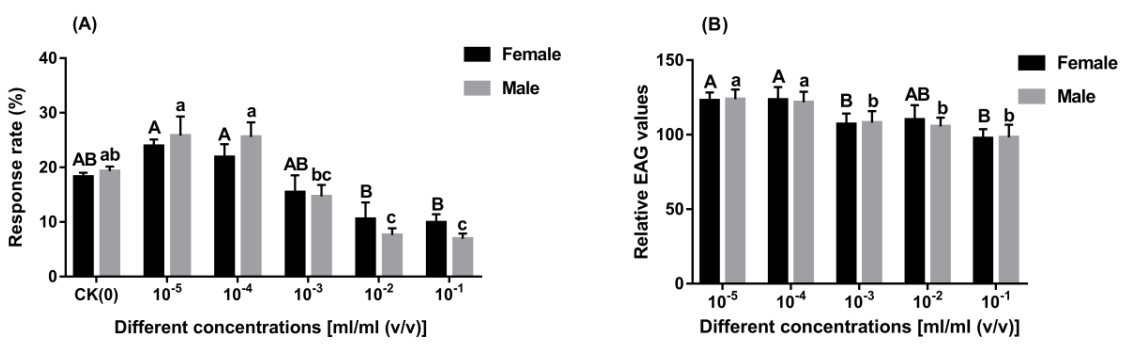

Figure 1. Behavioral (A) and EAG (B) dose-responses of $F$. arisanus to ethyl octanoate. The data are expressed as mean $\pm S E$. Different uppercase (female) or lowercase (male) letters indicate significant differences between concentrations (Tukey's HSD test after ANOVA, $P<0.05$ )
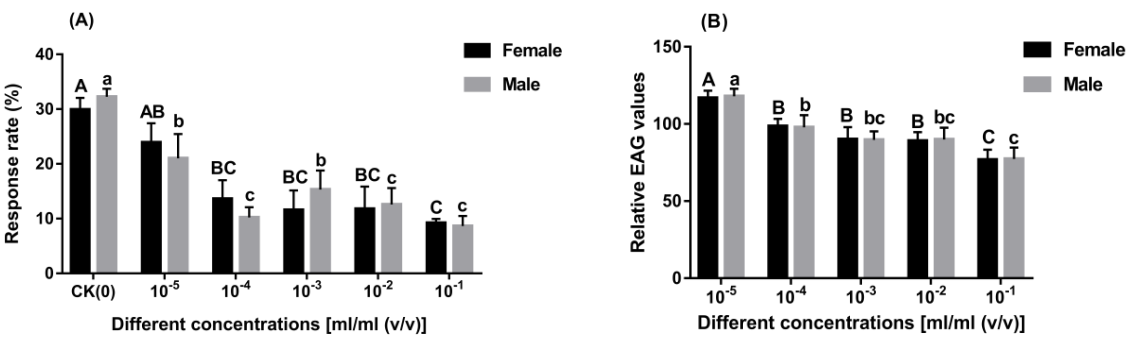

Figure 2. Behavioral $(A)$ and $E A G(B)$ dose-responses of $F$. arisanus to benzaldehyde. The data are expressed as mean $\pm S E$. Different uppercase (female) or lowercase (male) letters indicate significant differences between concentrations (Tukey's HSD test after ANOVA, $P<0.05$ )
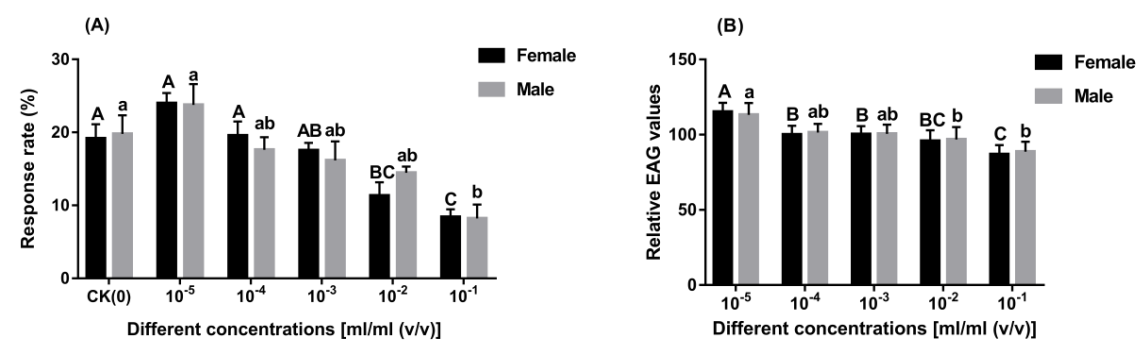

Figure 3. Behavioral (A) and EAG $(B)$ dose-responses of $F$. arisanus to methyl octanoate. The data are expressed as mean \pm SE. Different uppercase (female) or lowercase (male) letters indicate significant differences between concentrations (Tukey's HSD test after ANOVA, $P<0.05$ )
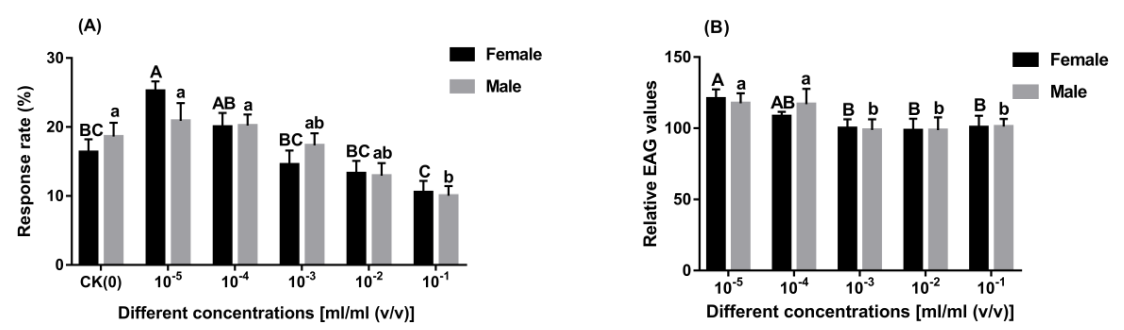

Figure 4. Behavioral $(A)$ and $E A G(B)$ dose-responses of $F$. arisanus to linalool. The data are expressed as mean \pm SE. Different uppercase (female) or lowercase (male) letters indicate significant differences between concentrations (Tukey's HSD test after ANOVA, $P<0.05$ ) 

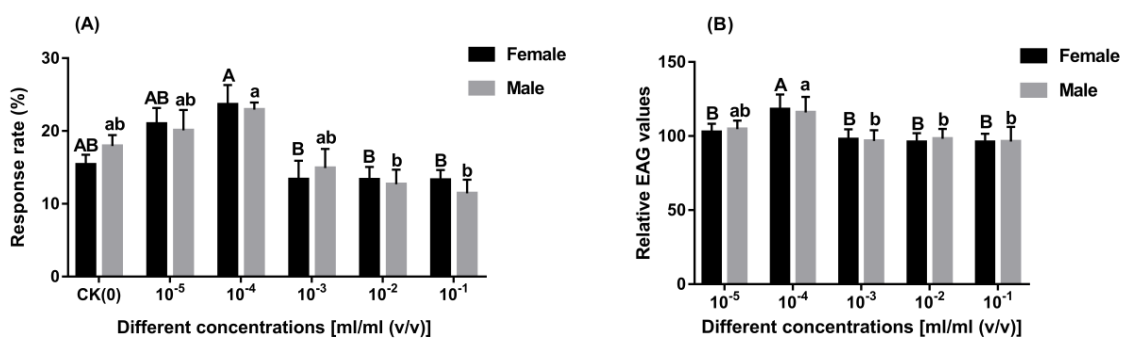

Figure 5. Behavioral $(A)$ and $E A G(B)$ dose-responses of $F$. arisanus to tetradecane. The data are expressed as mean $\pm S E$. Different uppercase (female) or lowercase (male) letters indicate significant differences between concentrations (Tukey's HSD test after ANOVA, $P<0.05$ )
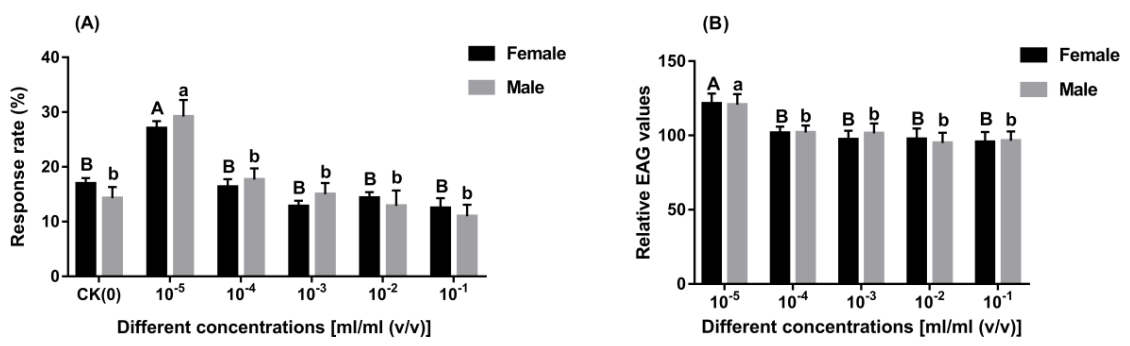

Figure 6. Behavioral $(A)$ and $E A G(B)$ dose-responses of $F$. arisanus to pentadecane. The data are expressed as mean $\pm S E$. Different uppercase (female) or lowercase (male) letters indicate significant differences between concentrations (Tukey's HSD test after ANOVA, $P<0.05$ )
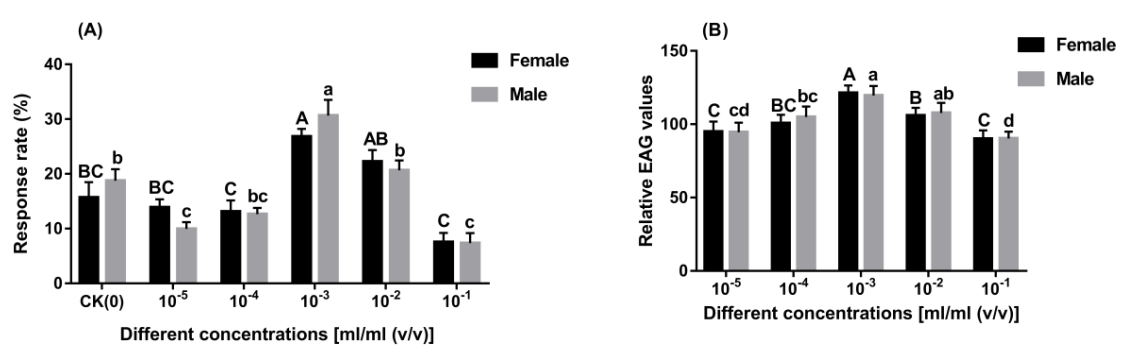

Figure 7. Behavioral (A) and EAG (B) dose-responses of F. arisanus to cis-3-hexenyl acetate. The data are expressed as mean $\pm S E$. Different uppercase (female) or lowercase (male) letters indicate significant differences between concentrations (Tukey's HSD test after ANOVA, $P<0.05$ )
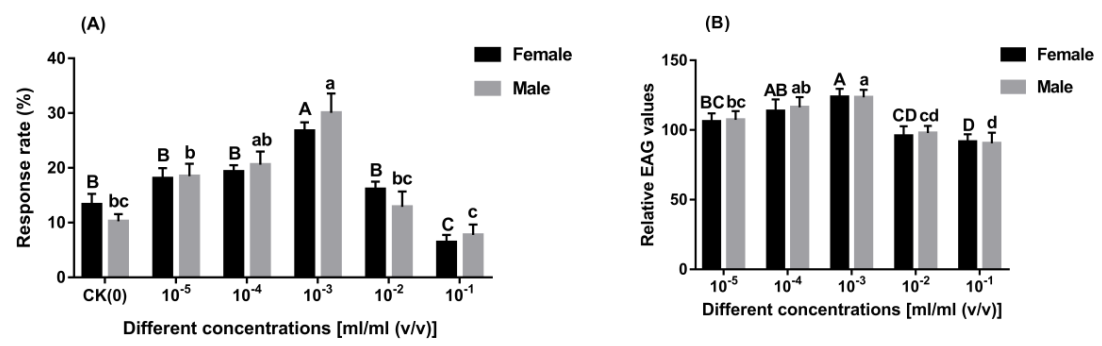

Figure 8. Behavioral $(A)$ and $E A G(B)$ dose-responses of $F$. arisanus females to $\beta$-myrcene. The data are expressed as mean $\pm S E$. Different letters indicate significant differences between concentrations (Tukey's HSD test after ANOVA, $P<0.05$ ) 

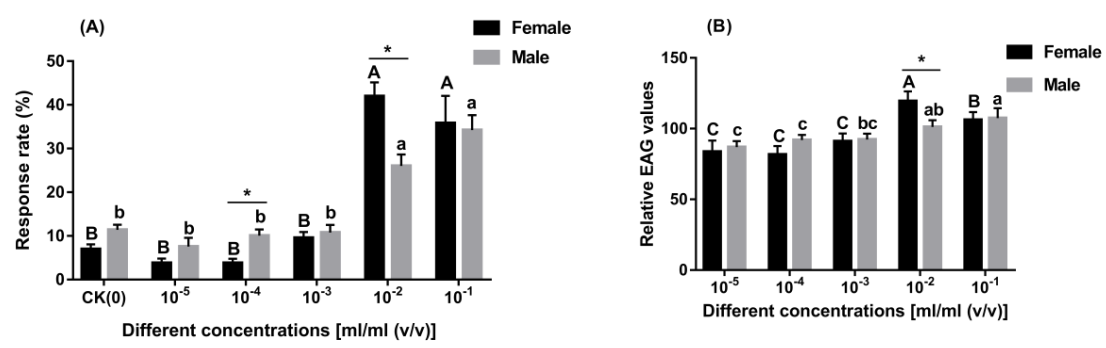

Figure 9. Behavioral (A) and $E A G(B)$ dose-responses of $F$. arisanus to trans farnesol. The data are expressed as mean \pm SE. Different uppercase (female) or lowercase (male) letters indicate significant differences between concentrations (Tukey's HSD test after ANOVA, P < 0.05). Asterisks indicate significant differences between females and males using an independent $t$-test at $P<0.05$
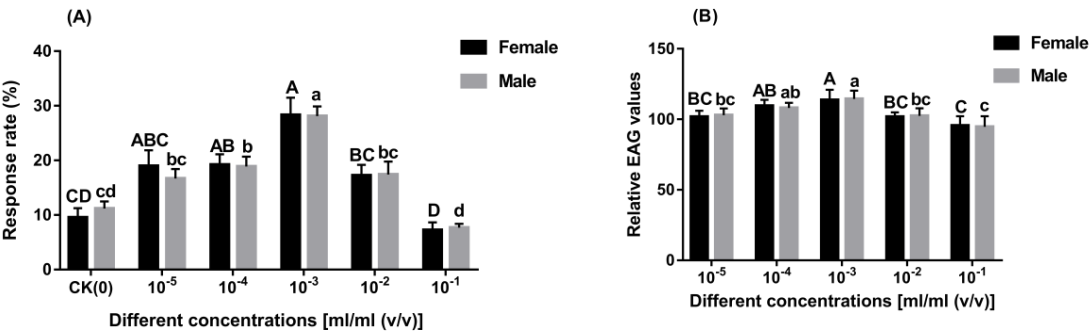

Figure 10. Behavioral $(A)$ and $E A G(B)$ dose-responses of $F$. arisanus to $\beta$-ocimene. The data are expressed as mean $\pm S E$. Different uppercase (female) or lowercase (male) letters indicate significant differences between concentrations (Tukey's HSD test after ANOVA, $P<0.05$ )

\section{Steady increase}

Response rates and EAG relative values increased as the dose increased and peaked at the highest concentration. This applied only to trans farnesol (Fig. 9).

\section{Inverted $V$}

Response rates and EAG relative values increased as the doses increased and peaked at a specific dose, then reduced as the dose further increased. The group comprised the results for cis-3-hexenyl acetate, $\beta$-myrcene and $\beta$-ocimene (Figs. 7, 8 and 10).

\section{Fluctuating}

Only one trend was recorded as having this type: the response rates of $F$. arisanus males to benzaldehyde which first decreased, then increased, then decreased as the concentration increased (Fig. 2A).

\section{Differences between sexes in their behavioral and antennal responses}

In most cases, there were no significant differences in either the EAG relative values or the response rates of parasitoids between males and females at each concentration. However, for trans farnesol significant differences in response rates were observed at concentrations of $10^{-4}(F=0.007, t=3.892, P=0.004)$ and $10^{-2} \mathrm{ml} / \mathrm{ml}(v / v)(F=2.230$, 
$t=-3.683, P=0.004)($ Fig. $9 A)$ and for the EAG relative values at $10^{-2} \mathrm{ml} / \mathrm{ml}(v / v)$ $(F=0.005, t=-2.749, P=0.021)(F i g .9 B)$.

Table 2. Concentration threshold $(\mathrm{ml} / \mathrm{ml})$ of different components that trigger the statistically highest response rate and $E A G$ relative values of $F$. arisanus

\begin{tabular}{|c|c|c|c|c|c|c|c|c|}
\hline \multirow{3}{*}{ Chemicals } & \multicolumn{4}{|c|}{ Behavioral response } & \multicolumn{4}{|c|}{ EAG relative values } \\
\hline & \multicolumn{2}{|c|}{ Female } & \multicolumn{2}{|c|}{ Male } & \multicolumn{2}{|c|}{ Female } & \multicolumn{2}{|c|}{ Male } \\
\hline & Threshold & $F$ and $P$ & Threshold & $F$ and $P$ & Threshold & $F$ and $P$ & Threshold & $F$ and $P$ \\
\hline Benzaldehyde & $0-10^{-5}$ & $\begin{array}{l}F=7.061, \\
P<0.001\end{array}$ & 0 & $\begin{array}{l}F=9.272, \\
P<0.001\end{array}$ & $10^{-5}$ & $\begin{array}{c}F=30.163 \\
P<0.001\end{array}$ & $10^{-5}$ & $\begin{array}{c}F=24.399, \\
P<0.001\end{array}$ \\
\hline Ethyl octanoate & $0-10^{-3}$ & $\begin{array}{l}F=6.992 \\
P<0.001\end{array}$ & $0-10^{-4}$ & $\begin{array}{c}F=15.802 \\
P<0.001\end{array}$ & $10^{-5}-10^{-4}, 10^{-2}$ & $\begin{array}{c}F=10.801 \\
P<0.001\end{array}$ & $10^{-5}-10^{-4}$ & $\begin{array}{c}F=11.532, \\
P<0.001\end{array}$ \\
\hline Methyl octanoate & $0-10^{-3}$ & $\begin{array}{c}F=13.277 \\
P<0.001\end{array}$ & $0-10^{-2}$ & $\begin{array}{c}F=5.746, \\
P=0.001\end{array}$ & $10^{-5}$ & $\begin{array}{c}F=13.794 \\
P<0.001\end{array}$ & $10^{-5}-10^{-3}$ & $\begin{aligned} F & =7.913, \\
P & =0.001\end{aligned}$ \\
\hline Linalool & $10^{-5}-10^{-4}$ & $\begin{array}{c}F=8.506, \\
P<0.001\end{array}$ & $0-10^{-2}$ & $\begin{array}{l}F=5.120, \\
P=0.002\end{array}$ & $10^{-5}-10^{-4}$ & $\begin{array}{c}F=9.645, \\
P<0.001\end{array}$ & $10^{-5}-10^{-4}$ & $\begin{aligned} F & =7.142, \\
P & =0.001\end{aligned}$ \\
\hline Tetradecane & $0-10^{-4}$ & $\begin{array}{l}F=4.891, \\
P=0.002\end{array}$ & $0-10^{-3}$ & $\begin{array}{l}F=4.708, \\
P=0.003\end{array}$ & $10^{-4}$ & $\begin{array}{c}F=8.905, \\
P<0.001\end{array}$ & $10^{-5}-10^{-4}$ & $\begin{array}{l}F=5.093, \\
P<0.001\end{array}$ \\
\hline Pentadecane & $10^{-5}$ & $\begin{array}{c}F=17.590 \\
P<0.001\end{array}$ & $10^{-5}$ & $\begin{array}{c}F=7.483 \\
P<0.001\end{array}$ & $10^{-5}$ & $\begin{array}{c}F=14.349 \\
P<0.001\end{array}$ & $10^{-5}$ & $\begin{array}{c}F=13.000, \\
P<0.001\end{array}$ \\
\hline $\begin{array}{l}\text { cis-3-hexenyl } \\
\text { acetate }\end{array}$ & $10^{-3}-10^{-2}$ & $\begin{array}{c}F=12.120, \\
P<0.001\end{array}$ & $10^{-3}$ & $\begin{array}{c}F=19.917 \\
P<0.001\end{array}$ & $10^{-3}$ & $\begin{array}{c}F=21.630 \\
P<0.001\end{array}$ & $10^{-3}-10^{-2}$ & $\begin{array}{c}F=16.451, \\
P<0.001\end{array}$ \\
\hline$\beta$-myrcene & $10^{-3}$ & $\begin{array}{c}F=18.641, \\
P<0.001\end{array}$ & $10^{-4}-10^{-3}$ & $\begin{array}{c}F=10.917 \\
P<0.001\end{array}$ & $10^{-4}-10^{-3}$ & $\begin{array}{c}F=19.490 \\
P<0.001\end{array}$ & $10^{-4}-10^{-3}$ & $\begin{array}{c}F=21.823, \\
P<0.001\end{array}$ \\
\hline Trans-farnesol & $10^{-2}-10^{-1}$ & $\begin{array}{c}F=32.970 \\
P<0.001\end{array}$ & $10^{-2}-10^{-1}$ & $\begin{array}{c}F=24.057, \\
P<0.001\end{array}$ & $10^{-2}$ & $\begin{array}{c}F=31.240 \\
P<0.001\end{array}$ & $10^{-2}-10^{-1}$ & $\begin{array}{c}F=14.070, \\
P<0.001\end{array}$ \\
\hline
\end{tabular}

\section{Discussion}

Plants can alter the emission of their volatile profile in response to herbivore foraging or pest oviposition (Hilker and Meiners, 2011). The selective exploration of HIPVs by parasitoids when searching their phytophagous hosts has been recorded on different parasitoids species (Dicke and van Loon, 2000), concentrated on those that parasitize folivorous larvae. Recently, various methods of parasitoid-based pest control have been developed that incorporate the use of volatile infochemicals as part of non-toxic, lowcost and effective strategies (Pickett et al., 2014). Infochemicals are disseminated through plants as a defense mechanism to directly and/or indirectly protect themselves from infestation by pests (Khan et al., 2010). For example, foraging parasitoids use HIPVs (a type of infochemicals) as reliable cues at both long- and short-range to locate their hosts in nature; this effectively protects the infested plant from their pests (NgiSong et al., 2000). Numerous studies have demonstrated that infestation by tephritid fruit flies, including $B$. dorsalis, enhances the attraction of $F$. arisanus adults to diverse crops species (Rousse et al., 2007b; Pérez et al., 2013; Ayelo et al., 2017; Gu et al., 2017). Thus, using infochemicals from the host habitats of pests will help design and enhance parasitoid-based biological control programs. It is essential to evaluate which volatile composition are identified at the olfactory level along with the minimum dosages required to evoke behavioral responses in a particular parasitoid (James, 2005).

Our research group has previously qualitatively and quantitatively compared the volatile profiles of four kinds of host crops before and after oviposition by $B$. dorsalis. 
The results suggested that ten compounds were exclusive to infested crops compared to healthy ones, and are generated by the host crop rather than the pest (Gu et al., 2017; Ji et al., 2016). Indeed, we concluded that guava fruits release benzaldehyde, cis-3hexenyl acetate, tetradecane, pentadecane and trans farnesol, and that citrus fruit release ethyl octanoate, methyl octanoate, pentadecane, $\beta$-ocimene, linalool and $\beta$-myrcene in response to infestation by $B$. dorsalis ( $\mathrm{Gu}$ et al., 2017). The emissions of most of these HIPVs is possibly attributed to the infested crop's active defense response because they are only detected after herbivore infestation and they are not emitted from healthy fruit, which is important in the host location process of parasitoids. Our present study revealed that these ten HIPV components, at different concentrations, can elicit different levels of electrophysiological and behavioral response in both sexes of $F$. arisanus. This provides yet more evidence that $F$. arisanus uses chemical cues derived from host crops, in concordance with speculations by other authors (Altuzar et al., 2004; Ayelo et al., 2017)

Insects are equipped with a highly sensitive olfactory system that enable them to probe and distinguish related volatile infochemicals with a high level of specificity and selectivity (Bruce and Pickett, 2011). By using electrophysiological and behavioral studies, it is possible to identify potentially bioactive volatile compounds and establish their biological function in parasitoids (Webster et al., 2010). In the present research, behavioral choice and EAG assays in $F$. arisanus confirmed 10 synthetic HIPVs to be biologically active. It is worth noting that among these volatile components, many single components have been demonstrated as eliciting positive behavioral or/and EAG responses in other braconids parasitoids [e.g. linalool for Cotesia marginiventris Cresson (Ngumbi and Fadamiro, 2012), ethyl octanoate for Psyttalia concolor Szépligeti (Benelli et al., 2013), benzaldehyde for Lysiphlebia japonica Ashmead (Hou et al., 2008), tetradecane and cis-3-hexenyl acetate for Microplitis croceipes Cresson (Morawo et al., 2016; Chen and Fadamiro, 2007), $\beta$-myrcene and $\beta$-ocimene for Aphidius ervi Haliday (Corrado et al., 2007; Takemoto and Takabayashi, 2015) (Hymenoptera: Braconidae)] or in other hymenopteran parasitoids [e.g. pentadecane for Trichogramma exiguum Pinto and Platner (Paul et al., 2002) (Hymenoptera: Trichogrammatidae) and trans farnesol for Diaeretiella rapae M'Intosh (Hymenoptera: Aphidiidae)] (Reed et al., 1995).

The behavioral and EAG dose-response curves of the ten compounds studied in the present research can be divided into four types: steady decrease, steady increase, inverted V and fluctuating. Regarding the group "steady decrease", the strongest response was obtained at the lowest dose, whereas for "steady increase", this was obtained at the highest dose. We therefore speculated that $F$. arisanus may have a dosage threshold for perceiving different components which can trigger corresponding levels of behavioral responses and physiological activities. For example, if the dosage is lower than the threshold value, the olfactory sensitivity of the parasitoids would elevate as the dose increased; however, if the dosage is higher than the threshold, sensitivity would descend as the dose increased. According to this, the concentration threshold of the volatile components that belonged to the group "inverted V" could be easily determined, i.e. cis-3-hexenyl acetate, $\beta$-ocimene and $\beta$-myrcene at $10^{-3} \mathrm{ml} / \mathrm{ml}(v / v)$. The step-like ability of benzaldehyde to lure $F$. arisanus was observed at our tested concentrations. Hiroyuki and Junji (2015) also reported a similar response in a braconid parasitoid to a volatile substance: using a Y-tube olfactometer, they found that $A$. ervi females, an effective natural enemy of several aphid species, were significantly attracted 
to $\alpha$ - phellandrene at doses of 30 and $0.1 \mathrm{ng}$, but not at 10- and 1- ng. Both our findings and those of Hiroyuki and Junji (2015) indicate that HIPVs can carry information at a variety of dose ranges, instead of being restricted to a single optimal dose range. The reason why $F$. arisanus has evolved to respond to two dose ranges in their host location behavior warrants further investigation.

The behavioral dose-response curves of volatile substances belonging to the "steady decrease" group are of particular interest. Of these compounds, only benzaldehyde at a concentration of $10^{-5} \mathrm{ml} / \mathrm{ml}(\mathrm{v} / \mathrm{v})$ attracted significantly fewer parasitoids compared to the control group, whereas in contrary to other compounds in this group. This suggested that benzaldehyde at our tested concentrations may impose a dose-dependent repellant effect on $F$. arisanus. The ecological role of benzaldehyde on parasitoids deserves further investigation. Regarding the differences between sexes, both the behavioral and EAG responses exhibited similar trends. Only one compound showed a significant difference, i.e. trans farnesol at $10^{-4}$ and $10^{-2} \mathrm{ml} / \mathrm{ml}(v / v)$ for behavioral responses and $10^{-2} \mathrm{ml} / \mathrm{ml}(v / v)$ for EAG responses. We hypothesize that both female and male $F$. arisanus may utilize the same olfactory receptors to perceive the other nine infochemicals (although with varying sensitivity), whilst having distinct sensing mechanisms for trans farnesol. In parasitoid-based biological control programs, the female has a vital role in pest suppression, directly resulting in the death of pests (Mills and Getz, 1996); thus, trans farnesol may be a more valuable volatile component.

In summary, HIPVs are considered as kairomones for several parasitoid species (Carrasco et al., 2005; Dweck et al., 2010; Benelli et al., 2013) and have already been successfully applied in the field (Uefune et al., 2012). However, detailed knowledge concerning the effect of HIPVs on the host-seeking behavior of biological control agents in the field requires further investigation prior to any possible commercial utilization (Kaplan, 2012). Besides recruiting beneficial arthropods, we believe that suitable HIPV candidates for field application should also possess additional useful features: (1) improving the defensive activities of intrinsic or imported biological control agents; (2) repelling pests or impose negative effects on the colonization and development of damaging pests; (3) exerting positive effects on the crop of economic importance; (4) without any adverse effects on non-target organisms or environment. For example, methyl salicylate at a certain concentration can not only attract Aphidius gifuensis Ashmead females (Hymenoptera: Braconidae) (Song, 2019), but can also inhibit the colonization and development of Myzus persicae Sulzer (Hemiptera: Aphididae) on tobacco leaves and provide a repellant effect (Liu et al., 2013). Furthermore, this substance can improve the parasitism performance of Anagrus nilaparvatae Pang et Wang (Hymenoptera: Mymaridae) towards eggs of Nilaparvata lugens Stal (Homoptera: Delphacidae) eggs (Wang and Lou, 2013).

Outside of direct field application, HIPVs also could be applied to enhance the effectiveness of mass-rearing beneficial insects since these compounds can improve the parasitization performance on alternative hosts. In another use, the host-seeking ability of $F$. arisanus females could be augmented by associative learning using the bioactive volatile compounds: the compounds could be incorporated into the diet of parasitoids at different phases and/or through a "reward" strategy before field release. As such, HIPVs with multiple positive ecological roles could achieve many things simultaneously, thus enhancing the efficiency of a series of plant protection strategies. Accurately characterizing these chemicals, along with investigating their potential in a variety of applications, is therefore of great value in integrated pest management. 


\section{Conclusion}

Infochemicals are produced from either the herbivorous host or its habitat and are used by parasitoids use to search for their hosts. A greater understanding of the tritrophic context of fruit - fruit fly - parasitoid systems will help improve the effectiveness of biocontrol programs that use parasitoids against destructive pests. In the present study, our results demonstrated that individually applied HIPVs can elicit different levels of antennal and behavioral responses from both sexes of $F$. arisanus when used at a variety of concentrations. This indicates that the tested components could be utilized to modulate parasitoid behavior, at least under laboratory conditions. However, thus far, the field application of a specific infochemical has only been reported for the pest sex pheromone, where it was used to improve parasitoid foraging activity. The use of infochemicals in pest control therefore appears to be a desirable tactic and thus we strongly recommend that field investigations are performed to both evaluate their practical effects in nature and comprehensively understand their ecological roles. We hope that the findings of the present study will help to screen for appropriate HIPV components, and enhance biocontrol efforts in sustainably suppressing fruit fly populations.

Acknowledgements. We thank Dr. Yanchuan Yang for helping to conduct part of the bioassay, and Ms. Shumei Wang for helping to rear insects. This research was financially supported by Industry University Research Project of Fujian Science and Technology Department (2019N5003),

Advanced Talents Introduction Project of Wuyi University (YJ201910), Education and Scientific Research Project for Young and Middle-aged Teachers in Fujian Province (JAT190801, JAT190805), National Key R\&D Program of China (2017YFD0201008) and Scientific Research Team Jointly Build By Teachers and Students of Wuyi University (for Chunhua Ma). We are also grateful to Mr. Alexander Barton of englishedit.co.uk for editing the English language of our manuscript.

Conflict of interests. The authors declare that they have no conflicts of interest. The funders had no role in the design of the study; in the collection, analyses, or interpretation of data; in the writing of the manuscript, or in the decision to publish the results.

\section{REFERENCES}

[1] Altuzar, A., Montoya, P., Rojas, J. C. (2004): Response of Fopius arisanus (Hymenoptera: Braconidae) to fruit volatiles in a wind tunnel. - Florida Entomologist 87: 616-618.

[2] Ayelo, P. M., Sinzogan, A. A., Bokonon-Ganta, A. H., Karlsson, M. F. (2017): Host species and vegetable fruit suitability and preference by the parasitoid wasp Fopius arisanus. - Entomologia Experimentalis et Applicata 163: 70-81.

[3] Bautista, R. C., Harris, E. J. (1996): Effect of fruit substrates on parasitization of tephritid fruit flies (Diptera) by the parasitoid Biosteres arisanus (Hymenoptera: Braconidae). Environmental Entomology 25: 470-475.

[4] Bautista, R. C., Harris, E. J., Vargas, R. I., Jang, E. B. (2004): Parasitization of melon fly (Diptera: Tephritidae) by Fopius arisanus and Psyttalia fletcheri (Hymenoptera: Braconidae) and the effect of fruit substrates on host preference by parasitoids. Biological Control 30: 156-164.

[5] Benelli, G., Revadi, S., Carpita, A., Giunti, G., Raspi, A., Anfor, G., Canale, A. (2013): Behavioral and electrophysiological responses of the parasitic wasp Psyttalia concolor (Szépligeti) (Hymenoptera: Braconidae) to Ceratitis capitata-induced fruit volatiles. Biological Control 64: 116-124. 
[6] Bruce, T. J. A., Pickett, J. A. (2011): Perception of plant volatile blends by herbivorous insects - finding the right mix. - Phytochemistry 72: 1605-1611.

[7] Carrasco, M., Montoya, P., Cruz-Lopez, L., Rojas, J. C. (2005): Response of the fruit fly parasitoid Diachasmimorpha longicaudata (Hymenoptera: Braconidae) to mango fruit volatiles. - Environmental Entomology 34: 576-583.

[8] Chen, L., Fadamiro, H. Y. (2007): Differential electroantennogram response of females and males of two parasitoid species to host-related green leaf volatiles and inducible compounds. - Bulletin of Entomological Research 97: 515-522.

[9] Corrado, G., Sasso, R., Pasquariello, M., Iodice, L., Carretta, A., Cascone, P., Ariati, L., Digilio, M. C., Guerrieri, E., Rao, R. (2007): Systemin regulates both systemic and volatile signaling in tomato plants. - Journal of Chemical Ecology 33: 669-681.

[10] Degenhardt, J., Köllner, T. G., Gershenzon, J. (2009): Monoterpene and sesquiterpene synthases and the origin of terpene skeletal diversity in plants. - Phytochemistry 70: 1621-1637.

[11] Dicke, M., van, Loon, J. J. A. (2000): Multitrophic effects of herbivore-induced plant volatiles in an evolutionary context. - Entomologia Experimentalis et Applicata 97: 237249.

[12] Dweck, H. K. M., Svensson, G. P., Gündüz, E. A., Anderbrant, O. (2010): Kairomonal response of the parasitoid Bracon hebetor Say to the male-produced sex pheromone of its host the greater waxmoth Galleria mellonella. - Journal of Chemical Ecology 36: 171178.

[13] Godfray, H. C. J. (1994): Parasitoids: Behavioral and Evolutionary Ecology. - Princeton University Press, Princeton.

[14] Gu, X. H., Cai, P. M., Yang, Q. Y., Ji, Q. E., Chen, J. H. (2017): Behavioral responses of Fopius arisanus (Sonan) (Hymenoptera: Braconidae) to volatiles from fruits infested by Bactrocera dorsalis (Hendel) (Diptera: Tephritidae) and analysis of volatile components. - Journal of Environmental Entomology 39: 820-829.

[15] Gu, X. H., Cai, P. M., Yang, Y. C., Yang, Q. Y., Yao, M. Y., Idrees, A., Ji, Q. E., Yang, J. Q., Chen, J. H. (2018): The response of four braconid parasitoid species to methyl eugenol: Optimization of a biocontrol tactic to suppress Bactrocera dorsalis. - Biological Control 122: 101-108.

[16] Harris, E. J., Bautista, R. C. (1996): Effects of fruit fly host, fruit species, and host egg to female parasitoid ratio on the laboratory rearing of Biosteres arisanus. - Entomologia Experimentalis et Applicata 79: 187-194.

[17] Harris, E. J., Bautista, R. C., Spencer, J. P. (2000): Utilization of the Egg-Larval Parasitoid, Fopius (Biosteres) arisanus, for Augmentative Control of Tephritid Fruit Flies. - In: Tan, K. H. (ed.) Area-Wide Control of Fruit Flies and Other Insect Pests. Penerbit Universiti Sains Malaysia, Penang, Malaysia.

[18] Hilker, M., Meiners, T. (2011): Plants and insect eggs: How do they affect each other? Phytochemistry 72: 1612-1623.

[19] Hou, Z. Y., Yan, F. S., Chen, X. (2008): Olfactory responses of Lysiphlebia japonica to volatile chemicals and fresh leaves of the host plants of cotton aphids in olfactometer. Insect Science 3: 49-57.

[20] James, D. G. (2005): Further field evaluation of synthetic herbivore-induced plant volatiles as attractants for beneficial insects. - Journal of Chemical Ecology 31: 481-495.

[21] Ji, Q. E., Bi, K., Chen, J. H. (2016): Response of egg-pupal parasitoid Fopius arisanus (Sonan) to infochemicals from the host eggs' surface of Bactrocera dorsalis (Hendel). Journal of Asia-Pacific Entomology 19: 1151-1157.

[22] Kaplan, I. (2012): Attracting carnivorous arthropods with plant volatiles: the future of biocontrol or playing with fire? - Biological Control 60: 77-89.

[23] Khan, Z. R., Midega, C. A. O., Bruce, T. J. A., Hooper, A. M., Pickett, J. A. (2010): Exploiting phytochemicals for developing a 'push-pull' crop protection strategy for cereal farmers in Africa. - Journal of Experimental Botany 61: 4185-4196. 
[24] Liquido, N. J. (1991): Effect of ripeness and location of papaya fruits on the parasitization rates of Oriental fruit fly and melon fly (Diptera: Tephritidae) by braconid (Hymenoptera) parasitoids. - Environmental Entomology 20: 1732-1736.

[25] Liu, T., Li, W. Z., You, X. F., Chai, X. L., Fu, G. X., Yuan, G. H. (2013): Repellent and anti-settling activity of common plant-derived volatiles on Myzus persicae (Sulzer). Acta Tabacaria Sinica 19: 77-84.

[26] Manoukis, N., Geib, S., Seo, D., McKenney, M., Vargas, R. I., Jang, E. (2011): An optimized protocol for rearing Fopius arisanus, a parasitoid of tephritid fruit flies. Journal of Visualized Experiments (53): e2901.

[27] Mills, J., Getz, W. M. (1996): Modelling the biological control of insect pests: a review of host-parasitoid models. - Ecological Modelling 92: 121-143.

[28] Morawo, T., Burrows, M., Fadamiro, H. (2016): Electroantennogram response of the parasitoid Microplitis croceipes to host-related odors: The discrepancy between relative abundance and level of antennal responses to volatile compound. - F1000Research 5: 2725.

[29] Ngi-Song, A. J., Njagi, P. G. N., Torto, B., Overholt, W. A. (2000): Identification of behaviourally active components from maize volatiles for the stemborer parasitoid Cotesia flavipes Cameron (Hymenoptera: Braconidae). - International Journal of Tropical Insect Science 20: 181-189.

[30] Ngumbi, E., Fadamiro, H. (2012): Species and sexual differences in behavioural responses of a specialist and generalist parasitoid species to host-related volatiles. Bulletin of Entomological Research 102: 710-718.

[31] Paul, A. V. N., Singh, S., Singh, A. K. (2002): Kairomonal effect of some saturated hydrocarbons on the egg parasitoids Trichogramma brasiliensis (Ashmead) and Trichogramma exiguum Pinto \& Platner (Hymenoptera: Trichogrammatidae). - Journal of Applied Entomology 126: 409-416.

[32] Pérez, J., Rojas, J. C., Montoya, P., Liedo, P., Castillo, A. (2013): Anastrepha egg deposition induces volatiles in fruits that attract the parasitoid Fopius arisanus. - Bulletin of Entomological Research 103: 318-325.

[33] Pickett, J. A., Woodcock, C. M., Midega, C. A. O., Khan, Z. R. (2014): 'Push-pull' farming systems. - Current Opinion in Biotechnology 26: 125-132.

[34] Reed, H. C., Tan, S. H., Haapanen, K., Killmon, M., Elliott, N. C. (1995): Olfactory responses of the parasitoid Diaeretiella rapae (Hymenoptera: Aphidiidae) to odor of plants, aphids, and plant-aphid complexes. - Journal of Chemical Ecology 21: 407-418.

[35] Rousse, P., Chiroleu, F., Domerg, C., Quilici, S. (2007a): Naive Fopius arisanus females respond mainly to achromatic cues. - Biological Control 43: 41-48.

[36] Rousse, P., Chiroleu, F., Veslot, J., Quilici, S. (2007b): The host- and microhabitat olfactory location by Fopius arisanus suggests a broad potential host range. Physiological Entomology 32: 313-321.

[37] Schellhorn, N. A., Bianchi, F., Hsu, C. L. (2014): Movement of entomophagous arthropods in agricultural landscapes: links to pest suppression. - Annual Review of Entomology 59: 559-581.

[38] Schoonhoven, L. M., Van, Loon, J. J. A., Dicke, M. (2005): Insect-Plant Biology. Oxford University Press, Oxford.

[39] Song, Y. Z. (2019): The effects of tobacco volatiles on behavioral orientation of Aphidus gifuensis. - Master's Thesis, Fujian Agriculture and Forestry University.

[40] Takemoto, H., Takabayashi, J. (2015): Parasitic wasps Aphidius erviare more attracted to a blend of host-induced plant volatiles than to the independent compounds. - Journal of Chemical Ecology 41: 801-807.

[41] Uefune, M., Choh, Y., Abe, J., Shiojiri, K., Sano, K., Takabayashi, J. (2012): Application of synthetic herbivore-induced plant volatiles causes increased parasitism of herbivores in the field. - Journal of Applied Entomology 136: 561-567. 
[42] Vargas, R. I., Leblanc, L., Putoa, R., Eitam, A. (2007): Impact of introduction of Bactrocera dorsalis (Diptera: Tephritidae) and classical biological control releases of Fopius arisanus (Hymenoptera: Braconidae) on economically important fruit flies in French Polynesia. - Journal of Economic Entomology 100: 670-679.

[43] Vargas, R. I., Leblanc, L., Harris, E. J., Manoukis, N. C. (2012): Regional suppression of Bactrocera fruit flies (Diptera: Tephritidae) in the Pacific through biological control and prospects for future introductions into other areas of the world. - Insects 3: 727-742.

[44] Vargas, R. I., Stark, J. D., Banks, J., Leblanc, L., Manoukis, N. C., Peck, S. (2013): Spatial dynamics of two oriental fruit fly (Diptera: Tephritidae) parasitoids Fopius arisanus and Diachasmimorpha longicaudata (Hymenoptera: Braconidae) in a guava orchard in Hawaii. - Environmental Entomology 42: 888-901.

[45] Vet, L. E. M., Dicke, M. (1992): Ecology of infochemical use by natural enemies in a tritrophic context. - Annual Review of Entomology 37: 141-172.

[46] Vinatier, F., Tixier, P., Duyck, P. F., Lescourret, F. (2011): Factors and mechanisms explaining spatial heterogeneity: A review of methods for insect populations. - Methods in Ecology and Evolution 2: 11-22.

[47] Wang, P., Lou, Y. G. (2013): Screening and field evaluation of synthetic plant volatiles as attractants for Anagrus nilaparvatae Pang et Wang, an egg parasitoid of rice planthoppers. - Chinese Journal of Applied Entomology 50: 431-440.

[48] Webster, B., Bruce, T. J. A., Pickett, J. A., Hardie, J. (2010): Volatiles functioning as host cues in a blend become nonhost cues when presented alone to the black bean aphid. Animal Behaviour 79: 451-457. 